\title{
Rationale and Design of the STOP-OB Study for Evaluating the Effects of Tofogliflozin and Glimepiride on Fat Deposition in Type 2 Diabetes Patients Treated with Metformin/DPP-4 Inhibitor Dual Therapy
}

Hisamitsu Ishihara (D) - Motonobu Anai · Hiroaki Seino · Toru Kitazawa $\cdot$ Hiroshi Ohashi $\cdot$ Masumi Ai

Masahiro Inoue $\cdot$ Midori Fujishiro $\cdot$ Takeshi Inazawa $\cdot$ Hisamoto Kuroda $\cdot$ Masayo Yamada

Received: July 17, 2018 / Published online: August 25, 2018

(C) The Author(s) 2018

\section{ABSTRACT}

Background: The global pandemic of type 2 diabetes mellitus (T2DM) is an enormous clinical and socioeconomic burden. Biguanides and DPP-4 inhibitors (DPP-4i) are the most commonly used therapies in Japanese T2DM patients. When glycemic control is not adequate despite combination of these drugs, there is no consensus on the next step drug. Systematic reviews and meta-analyses of previous trials

Enhanced digital features To view enhanced digital features for this article go to https://doi.org/10.6084/ m9.figshare.6949829.

Electronic supplementary material The online version of this article (https://doi.org/10.1007/s13300018-0491-4) contains supplementary material, which is available to authorized users.

H. Ishihara $\cdot$ M. Fujishiro

Division of Diabetes and Metabolism, Nihon University School of Medicine, 30-1 Oyaguchi-

kamicho, Itabashi-ku, Tokyo 173-8610, Japan

H. Ishihara $(\varangle) \cdot$ M. Fujishiro

Department of Internal Medicine, Nihon University

Hospital, 1-6 Kanda-Surugadai, Chiyoda-ku,

Tokyo 101-8309, Japan

e-mail: ishihara.hisamitsu@nihon-u.ac.jp

M. Anai

Research Center for Advanced Science and

Technology, The University of Tokyo, 4-6-1

Komaba, Meguro-ku, Tokyo 153-8904, Japan have indicated that glycemic control with triple combination therapies yields similar results. Thus, beneficial effects on cardiovascular risk factors may be important. The present study was designed to evaluate body fat percentage and several insulin resistance parameters after addition of tofogliflozin or glimepiride to the regimens of patients being treated with metformin and a DPP-4 inhibitor but failing to attain adequate blood glucose control.

Methods: Sodium glucose cotransporter-2 inhibitor, tofogliflozin versus glimepiride, comparative trial in patients with type 2 diabetes on body composition is an ongoing, multicenter, prospective, randomized, open-label, parallel-group trial. T2DM patients treated with metformin/DPP-4 inhibitor dual therapy have been recruited and randomly assigned to $20 \mathrm{mg}$ /day tofogliflozin $(n=32)$ or $0.5 \mathrm{mg} /$ day

\author{
M. Anai \\ Division of Diabetes and Metabolism, The Institute \\ for Adult Diseases, Asahi Life Foundation, \\ 2-2-6 Nihonbashi Magui-cho, Chuo-ku, \\ Tokyo 103-0002, Japan \\ H. Seino \\ Seino Internal Medicine Clinic, 6-192-2 Kaisei, \\ Koriyama, Fukushima 963-8851, Japan \\ T. Kitazawa \\ Division of Diabetes, Endocrinology and \\ Metabolism, Tokyo Metropolitan Cancer and \\ Infectious Diseases Center Komagome Hospital, \\ 3-18-22 Honkomagome, Bunkyo-ku, \\ Tokyo 113-8677, Japan
}


glimepiride $(n=32)$ groups, with either of these drugs being added to pre-existing regimens for 24 weeks.

Planned Outcomes: The primary endpoint is the change in body fat percentage from baseline to 24 weeks. The secondary outcomes are changes in body composition other than fat percentage, body weight, parameters related to glycemic control and $\beta$-cell function, parameters related to lipids and arteriosclerosis, parameters related to liver function, parameters related to diabetic nephropathy, and uric acid levels. Safety parameters will also be analyzed. This is the first trial comparing the effects and safety of adding an SGLT2i and a sulfonylurea as the third-line oral agent to metformin/DPP-4i dual therapy. The results will provide valuable information for choosing third-line oral agents. Trial Registration: UMIN000026161.

Funding: Kowa Co. Ltd. and Kowa Pharmaceutical Co. Ltd., Tokyo, Japan.

Keywords: Fat deposition; SGLT2 inhibitor; Sulfonylurea; Third-line oral drug; Type 2 diabetes mellitus

$\begin{array}{ll}\text { Abbreviations } \\ \text { DPP-4 } & \text { Dipeptidylpeptidase-4 } \\ \text { FAS } & \text { Full analysis set } \\ \text { HbA1c } & \text { Glycated hemoglobin } \\ \text { OGTT } & \text { Oral glucose tolerance test } \\ \text { ODA } & \text { Oral diabetes agent } \\ \text { PPS } & \text { Per protocol set } \\ \text { SGLT2 } & \text { Sodium glucose cotransporter-2 }\end{array}$

H. Ohashi

Oyama East Clinic, 1-32-1 Ekihigashi-tori, Oyama, Tochigi 323-0022, Japan

M. Ai

Tanaka Clinic, 12-40 Hon-cho, Wako,

Saitama 351-0114, Japan

M. Ai

Department of Insured Medical Care Management, Graduate School of Medical and Dental Sciences,

Tokyo Medical and Dental University, 1-5-45

Yushima, Bunkyo-ku, Tokyo 113-8519, Japan

M. Inoue

Sasazuka Inoue Clinic, 1-15-4 Sasazuka, Shibuya-ku, Tokyo 151-0073, Japan
SU Sulfonylurea

T2DM Type 2 diabetes mellitus

\section{INTRODUCTION}

\section{Background}

Hyperglycemia in type 2 diabetes mellitus (T2DM) is caused by defects in the insulin secretory response from pancreatic $\beta$-cells and in insulin action on skeletal muscles, adipose tissues, and the liver. Impaired insulin action, or insulin resistance, is closely related to abdominal fat deposition and also accelerates cardiovascular complications. Patients with T2DM are being treated with either oral antidiabetic agents (OADs) or injectable agents, or both. Patients are often reluctant to start an injectable medication, since it may be painful and restrict their daily life activities. Japanese patients with T2DM are usually treated, after lifestyle modification, with either biguanides, sulfonylureas (SU), or DPP-4 inhibitors (DPP-4i) [1]. Biguanide and DPP-4i combination is the dual therapy most commonly used in Japan [2]. However, when glycemic control is not adequate despite these drugs, there is no consensus on the next step drug. Clinicians are advised to evaluate individual patients to ascertain the optimal therapy for each [3].

Sulfonylurea drugs, used for more than 50 years, stimulate insulin secretion and suppress glucose levels. The efficacy of SUs for

\footnotetext{
T. Inazawa

Department of Endocrinology and Metabolism, Kashiwa City Hospital, 1-3 Fuse, Kashiwa, Chiba 277-0825, Japan

H. Kuroda Green Clinic, 3-9-15 Midori-machi, Mibu, Tochigi 321-0204, Japan

M. Yamada

Yokohama Sakae Kyosai Hospital, 132 Katsura-cho, Sakae-ku, Yokohama, Kanagawa 247-8581, Japan
} 
controlling blood glucose is well recognized, and these agents are often used as the secondor third-line OAD, or even as the first drug $[1,2]$. Beneficial effects of combination therapy with a DPP-4 inhibitor have also been reported $[4,5]$. Furthermore, SUs are economical to use. However, SUs may cause hypoglycemia, especially when combined with a DPP-4i [6]. SUs may also cause weight gain, although there are reportedly inconsistent results when combined with metformin and/or a DPP-4i in Japanese patients [4]. It is noteworthy that the latest generation SU, glimepiride, may exert extrapancreatic effects enhancing insulin sensitivity [7-10].

Recently, sodium glucose cotransporter-2 inhibitors (SGLT2i) have emerged as promising treatments for T2DM. This class of drugs inhibits glucose reabsorption from urine, a mechanism independent of insulin action, which makes them appropriate for use with pre-existing agents. In addition, as a result of glucose loss from urine, body weight is often reduced when taking these drugs. They also exhibit pleiotropic effects on diabetic complications including heart failure, renal impairment, and hepatic dysfunction [11-13]. Tofogliflozin is an SGLT2i with 2900-fold greater selectivity for SGLT2 than SGLT1 and has the highest selectivity of all clinically developed inhibitors [14]. Randomized controlled studies in Japanese T2DM patients with inadequate glycemic control on diet/exercise therapy [15] or on other OADs [16] demonstrated that tofogliflozin significantly improved glycemic control and decreased body weight.

Several investigations have compared the efficacy and safety of an SU and an SGLT2i as second-line drugs after metformin: canagliflozin vs. glimepiride [17], empagliflozin vs. glimepiride [18], and dapagliflozin vs. glipizide [19]. SUs initially produced a greater drop in HbA1c but were essentially equivalent in efficacy to an SGLT2i after 52 weeks. Hypoglycemia was more frequent in the SU groups in all three trials, while genital infections were observed more frequently in the SGLT2i groups. Systematic reviews or meta-analyses of previous trials have indicated that glycemic control by triple combination therapy yielded similar results
[20-22]. Therefore, other merits besides glycemic control as well as disadvantages should be considered: for example, less hypoglycemia, less weight gain, and beneficial effects on cardiovascular risks. One of the prominent features of SGLT2i is the effects on cardiovascular complications [11-13]. Although the precise mechanisms by which SGLT2i exert beneficial effects on the cardiovascular system are unknown, diminished insulin resistance appears to play a role, which is closely related to reduced body weight and loss of fat. Although several reports have indicated body fat loss by SGLT2i [23-26] as possibly leading to improvement of insulin sensitivity, glimepiride has also been shown to have some effects on insulin sensitivity as well as possible extra-pancreatic effects [7-10].

\section{Objectives and Trial Design}

In this trial, therefore, we are attempting to compare the change in body fat percentage and insulin resistance parameters between patients, with either tofogliflozin or glimepiride added as the third-line agent, who have not achieved adequate glycemic control when treated with metformin and a DPP-4i. For this purpose, we have designed this multicenter, prospective, randomized, open-label, parallel-group trial.

\section{METHODS}

\section{Study Design}

Sodium glucose cotransporter-2 inhibitor, tofogliflozin versus glimepiride comparative trial in patients with type 2 diabetes on body composition (STOP-OB) is an ongoing, multicenter, prospective, randomized, open-label, parallelgroup trial. This trial was designed to compare tofogliflozin (20 mg/day) and glimepiride $(0.5 \mathrm{mg} /$ day $)$ as third-line oral agents on top of metformin and DPP-4is. Patients are randomized to either the tofogliflozin group or the glimepiride group (ratio 1:1) using the web-based minimization method for HbA1c level $(<8.0 \%$, $\geq 8.0 \%)$ and body mass index (BMI) $(<25.0$, $\left.\geq 25.0 \mathrm{~kg} / \mathrm{m}^{2}\right)$, at the time of providing 
informed consent. Following 24 weeks of treatment, clinical and biochemical data, including body composition, will be evaluated. All personal information and data will be anonymized.

This study has been registered on the University Hospital Medical Information Network Clinical Trials Registry (UMIN-CTR), which is a non-profit organization in Japan, and meets the requirements of the International Committee of Medical Journal Editors (ICMJE) (UMIN000026161).

The study protocol was approved by the institutional review board at each participating institution in compliance with the Declaration of Helsinki and current legal regulations in Japan. The study will be conducted in accordance with the Ethical Guidelines for Medical and Health Research Involving Human Subjects published by the Ministry of Health, Labor and Welfare of Japan and the Helsinki Declaration of 1964, as revised in 2013. Written informed consent for participation is to be obtained from all participants after a full explanation of the study has been provided by investigators. Any changes in the protocol will be submitted to the institutional review boards.

\section{Sample Selection}

Japanese T2DM subjects who periodically come to the outpatient clinics for diabetes at ten institutions in Japan (Supplementary Table 1) will be asked to participate in this study. The inclusion and exclusion criteria are as follows:

Inclusion criteria

1. T2DM patients treated with any of the DPP4is and metformin $(\geq 500$ and $\leq 1500 \mathrm{mg}$ /day) without dose changes for at least 4 weeks before the time of providing informed consent.

2. HbA1c levels of at least $7.0 \%$ but less than $9.0 \%$ at the time of providing informed consent.

3. Age $\geq 20$ and $<75$ years (regardless of gender).

4. Provision of written consent for participation in the study.

Exclusion criteria

1. Type 1 or secondary diabetes.
2. Use of antidiabetic drugs other than DPP4 is and metformin (including insulins and GLP-1 receptor agonists) 12 weeks prior to provision of consent.

3. Dose changes or start of medication of diuretics 12 weeks prior to provision of consent.

4. BMI less than $18.5\left(\mathrm{~kg} / \mathrm{m}^{2}\right)$ or at least 35 .

5. Contraindication or hypersensitivity to SGLT2is or SUs.

6. Moderate or severe renal dysfunction $\left(\mathrm{eGFR}<60 \mathrm{~mL} / \mathrm{min} / 1.73 \mathrm{~m}^{2}\right)$.

7. Severe liver dysfunction.

8. History of stroke or cerebral infarction.

9. For women, pregnant, lactating, possibly pregnant, or planning to become pregnant.

10. Moderate or severe heart failure (NYHA/ New York Heart Association stage III or more severe).

11. Urinary tract or genital infections.

12. Cancer or history of cancer (study inclusion possible if recurrence is confirmed to be absent).

13. Judged to be ineligible for any reason (such as steroid use or weight loss medication) by the clinical investigators.

\section{Treatment}

Participants treated with metformin and a DPP$4 \mathrm{i}$ are to be additionally given either tofogliflozin $(20 \mathrm{mg} /$ day) or glimepiride $(0.5 \mathrm{mg} /$ day $)$ after breakfast every day for 24 weeks. Treatment with tofogliflozin is to be followed by the official recommendation for appropriate use of SGLT2is from the Japan Diabetes Society. Each participant's regimen other than the study drugs will remain unchanged, with no drugs being either added or discontinued after provision of informed consent.

\section{Observation Items and Schedule}

The observation items and schedule are shown in Fig. 1. The study period is 24 weeks after the initial administration of the study drugs (registration period, January 2017-March 2018; full study duration, January 2017-December 2018). 


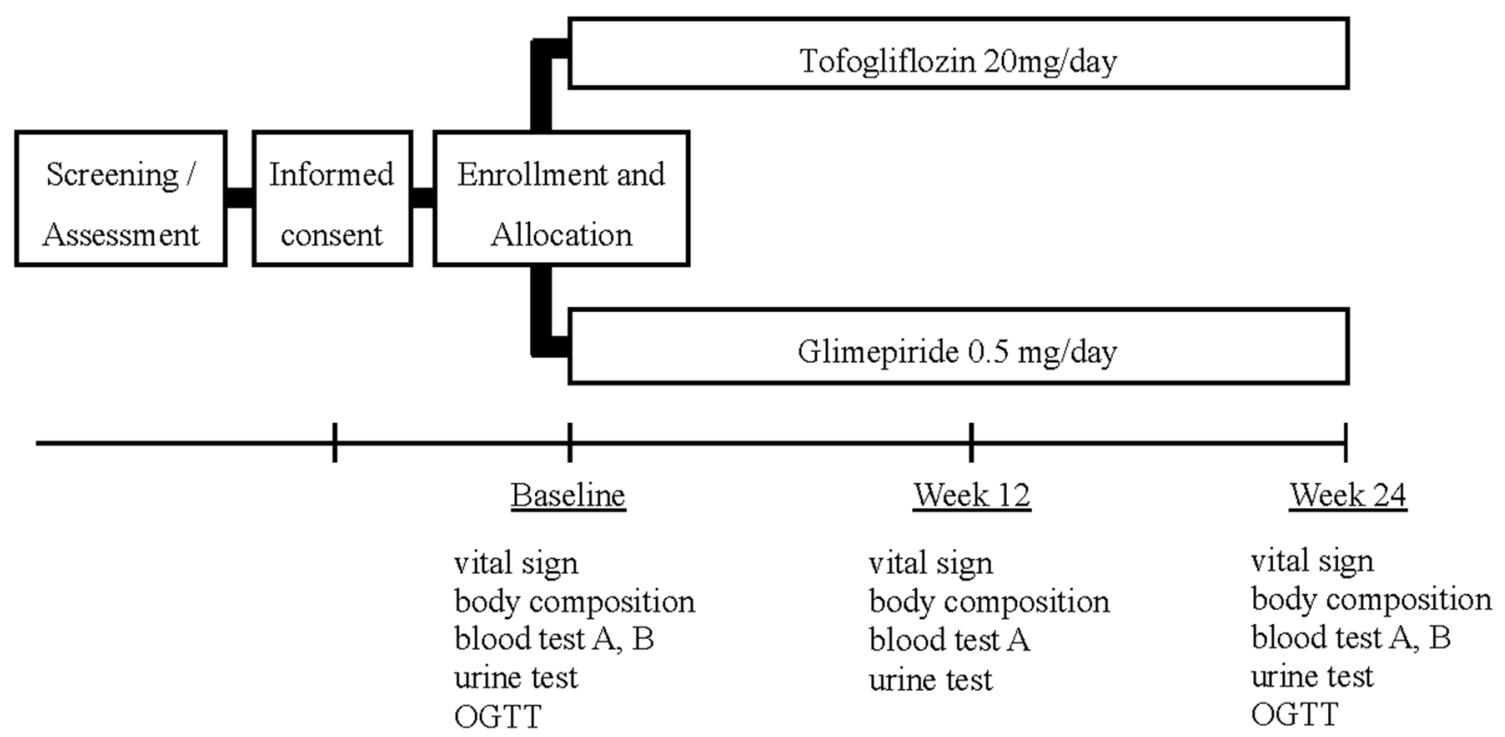

- vital sign: body weight, BMI, abdominal circumstance, blood pressure

- body composition: fat content, fat free-mass, muscle mass, total body water, total body water percentage, bone mass, basal metabolic rate, basal metabolic rate level, visceral fat rating, leg muscle rating, degree of obesity

- blood test A: HbA1c, TC, HDL-C, TG, LDL-C, serum creatinine, uric acid, AST, ALT, $\gamma$ GTP, type IV collagen $7 \mathrm{~S}^{*}$, ferritin**, hyaluronic acid* $^{*}$

- blood test B: adiponectin, leptin, ketone bodies

- urine test: urinary albumin*

- OGTT: plasma glucose, insulin, C-peptide, glucagon

* items with asterisks are optional test items.

Fig. 1 Study design

All randomized participants will be followed until the scheduled study completion, regardless of adherence to or discontinuation of study medication for any reason. The clinical outcomes, adherence, and adverse events will be ascertained, and clinical and biochemical data will be collected at 0,12 , and 24 weeks after randomization.

\section{Planned Outcomes}

The primary study outcome is the change in body fat percentage from baseline to 24 weeks. The secondary outcomes are changes in the following parameters from baseline to week 24 :
1. Body composition other than fat percentage (fat content, fat-free mass, muscle mass, total body water, total body water percentage, bone mass, basal metabolic rate (BMR), visceral fat rating, leg muscle score, and degree of obesity).

2. Body weight, BMI, abdominal circumference, and blood pressure.

3. Parameters related to glycemic control and $\beta$-cell function (HbA1c, plasma glucose, insulin, C-peptide, glucagon, HOMA- $\beta$, and HOMA-R).

4. Parameters related to lipids and arteriosclerosis (serum total cholesterol, HDL-cholesterol, triglyceride, LDL-cholesterol, adiponectin, and leptin). 
5. Parameters related to liver function (AST, ALT, $\gamma$ GTP, ketone bodies, type IV collagen 7S, ferritin, and hyaluronic acid).

6. Parameters related to diabetic nephropathy (serum creatinine, urinary albumin, and eGFR).

7. Serum uric acid.

8. Any adverse events, including hypoglycemia.

9. Drug adherence rates.

Body fat percentages, fat content, fat-free mass, muscle mass, total body water, total body water percentage, bone mass, BMR, BMR level, visceral fat rating, leg muscle rating, and degree of obesity will be measured using the dual cycle several composition DC-430A body composition analyzer (Tanita, Tokyo, Japan), which is the latest model after the DC-320 [27, 28]. Plasma glucose, insulin, C-peptide, and glucagon will be measured by $75 \mathrm{~g}$ oral glucose tolerance test (OGTT) at the following four measurement time points: before glucose intake (pre) and $0.5 \mathrm{~h}, 1 \mathrm{~h}$, and $2 \mathrm{~h}$ after glucose intake. The OGTT will be conducted with the subject in a fasted state at baseline and at week 24 . In the OGTT at week 24, participants will take their allocated study drugs after the pre-measurement and before glucose intake.

\section{Safety Evaluation}

The details and incidence of adverse events will be periodically ascertained. If the investigators identify any adverse events, the severity grades, procedures, outcomes, and relationships to the study drugs will be assessed and recorded on the case report form. For compensation to those who suffer harm from the study participation, clinical trial insurance will be provided.

\section{Sample Size}

A preceding study [17] found that body fat percentage changed in response to 52-week treatments with empagliflozin and glimepiride by $-0.6 \%$ and $1.1 \%$, respectively. We estimated changes in the body fat percentage after 24-week treatments with tofogliflozin and glimepiride to be $-0.6 \pm 2.2 \%$ and $1.1 \pm 2.2 \%$, respectively. On the basis of these estimations and with a two-sided significance level of 5\% and power of $80 \%$, the number of patients required to detect a significant difference in the change in the body fat percentage between the two groups was 28 patients per group, requiring a total sample size of 56 . Assuming a $10 \%$ dropout rate during the study, we set the target number of patients at 32 per group, i.e., a total of 64 patients.

\section{Data Analysis}

The primary and secondary outcomes, except for adverse events, will be analyzed using mainly the full analysis set (FAS) and the secondary per protocol set (PPS). The FAS includes all participants enrolled and randomized except for those with serious violations of the study protocol such as failing to provide informed consent and enrollment outside of the study period. The PPS includes participants excluded, as a result of any violation of the inclusion/exclusion criteria, use of prohibited drugs concomitantly, and poor compliance with the study drugs $(<75 \%, \geq 120 \%)$, from the FAS population. On the other hand, we will perform an analysis of adverse events using a safety analysis set, which will include all enrolled participants and those treated with any of the study agents.

The primary endpoint, the change in body fat percentage, will be analyzed using analysis of covariance models including the treatment group as the fixed effect and the baseline HbA1c level and BMI as covariances. In addition, as the sensitivity analysis, we plan to apply the mixed effects model for repeated measures including treatment group and time, the interaction between treatment group and time, the baseline HbA1c level and BMI as fixed effects, and with an unstructured covariance within-subject variability. For continuous variables of the secondary endpoints, we will perform Student's $t$ test for comparisons between groups, and the one-sample $t$ test for within-group changes. In the analysis of adverse events, Fisher's exact test will be applied for comparisons between groups. All statistical analyses will be two-tailed at a 
significance level of 0.05 , using SAS software version 9.4 (SAS Institute, Cary, NC, USA). The planned analysis will be described in detail in the statistical analysis plan that will be developed by the principal investigator and a biostatistician and fixed before locking of the database.

\section{Strengths and Limitations}

The strength of this trial is a multicenter, prospective, randomized, controlled, parallelgroup design. To the best of our knowledge, this is the first trial comparing the effects and safety of an SGLT2i and an SU added as the third-line oral agent to metformin/DPP-4i dual therapy. The limitations are the small number of participants and the open label trial design. In addition, although we consider the bio-impedance method to be satisfactory and convenient for measuring body fat mass but dual-energy X-ray absorptiometry, quantitative computer tomography, or magnetic resonance imaging might be an alternative.

\section{ACKNOWLEDGEMENTS}

The authors would like to thank patients participating in this study. We would also like to thank all of the staff in participating hospitals.

Funding. Financial support for this study including processing charges for any journal articles was also provided by Kowa Co. Ltd. and Kowa Pharmaceutical Co. Ltd., Tokyo, Japan. The funder has no role in study design, data collection and analysis, decision to publish or preparation of the manuscript. All authors had full access to all of the data in this study and take complete responsibility for the integrity of the data and accuracy of the data analysis.

Editorial Assistance. The authors gratefully acknowledge the assistance of $\mathrm{H}$ Yamada (Soiken Holdings Inc., Tokyo, Japan) for his editorial assistance, which was funded by Kowa Co. Ltd. and Kowa Pharmaceutical Co.
Authorship. All named authors meet the International Committee of Medical Journal Editors (ICMJE) criteria for authorship for this article, take responsibility for the integrity of the work as a whole, and have given their approval for this version to be published.

Authorship Contributions. Hisamitsu Ishihara, Motonobu Anai and Hiroaki Seino: conception and design of the study, enrollment and acquisition of data. Midori Fujishiro,Toru Kitazawa, Takeshi Inazawa, Masayo Yamada, Masahiro Inoue, Hiroshi Ohashi, Hisamoto Kuroda and Masumi Ai: enrollment and acquisition of data. All authors read and approved the final manuscript.

Disclosures. Hisamitsu Ishihara has served on the scientific advisory board of Astellas Pharma Inc.; received lecture or consulting fees from Astellas Pharma Inc., MSD, Sanofi, Mitsubishi Tanabe Pharma, Boehringer Ingelheim Japan, and Novartis Pharma; and has received grants/research support from Astellas Pharma Inc., Ono Pharmaceutical, Boehringer Ingelheim Japan, AstraZeneca, Sanofi, Mitsubishi Tanabe Pharma, Eli Lilly Japan, Daiichi-Sankyo, Novo Nordisk Pharma, Kyowa Hakko Kirin, and MSD. Motonobu Anai, Hiroaki Seino, Toru Kitazawa, Hiroshi Ohashi, Masumi Ai, Masahiro Inoue, Midori Fujishiro, Takeshi Inazawa, Hisamoto Kuroda and Masayo Yamada have nothing to disclose.

Compliance with Ethics Guidelines. The study protocol was approved by the institutional review board at each participating institution in compliance with the Declaration of Helsinki and current legal regulations in Japan. The study will be conducted in accordance with the Ethical Guidelines for Medical and Health Research Involving Human Subjects published by the Ministry of Health, Labor and Welfare of Japan and the Helsinki Declaration of 1964, as revised in 2013. Written informed consent for participation is to be obtained from all participants after a full explanation of the study has been provided by investigators. Any changes in the protocol will be submitted to the institutional review boards. 
Data Availability. The datasets generated and/or analyzed during this study will be available from the corresponding author upon reasonable request. The study results will be published in scientific journals.

Trial Status. The current protocol version is version1.1 created on November 20th 2016. Patient recruitment began on January 1st 2017 and ended on March 31st 2018.

Open Access. This article is distributed under the terms of the Creative Commons Attribution-NonCommercial 4.0 International License (http://creativecommons.org/licenses/ by-nc/4.0/), which permits any noncommercial use, distribution, and reproduction in any medium, provided you give appropriate credit to the original author(s) and the source, provide a link to the Creative Commons license, and indicate if changes were made.

\section{REFERENCES}

1. Kanatsuka A, Sato Y, Kawai K, et al. Relationship between the efficacy of oral antidiabetic drugs and clinical features in type 2 diabetic patients (JDDM38). J Diabetes Investig. 2016;7:386-95.

2. Tanabe M, Motonaga R, Terawaki Y, Nomiyama T, Yanase T. Prescription of oral hypoglycemic agents for patients with type 2 diabetes mellitus: a retrospective cohort study using a Japanese hospital database. J Diabetes Investig. 2017;8:227-34.

3. Edwards KL, Alvarez C, Irons BK, Fields J. Third-line agent selection for patients with type 2 diabetes mellitus uncontrolled with sulfonylureas and metformin. Pharmacotherapy. 2008;28:506-21.

4. Ishii H, Ohkubo Y, Takei M, et al. Efficacy of combination therapy with sitagliptin and low-dose glimepiride in Japanese patients with type 2 diabetes. J Clin Med Res. 2014;6:127-32.

5. Umayahara R, Yonemoto T, Kyou C, et al. Low-dose glimepiride with sitagliptin improves glycemic control without dose-dependency in patients with type 2 diabetes inadequately controlled on highdose glimepiride. Endocr J. 2014;61:1163-70.

6. Yamaguchi S, Ikejima M, Furukawa A, Abe M, Nakazaki M, Ishihara $H$. Octreotide for hypoglycemia caused by sulfonylurea and DPP-4 inhibitor. Diabetes Res Clin Pract. 2015;109:e8-10.

7. Tsunekawa T, Hayashi T, Suzuki Y, et al. Plasma adiponectin plays an important role in improving insulin resistance with glimepiride in elderly type 2 diabetic subjects. Diabetes Care. 2003;26:285-9.

8. Araki T, Emoto M, Konishi T, et al. Glimepiride increases high- density lipoprotein cholesterol via increasing adiponectin levels in type 2 diabetes mellitus. Metabolism. 2009;58:143-8.

9. Xu DY, Zhao SP, Huang QX, et al. Effects of glimepiride on metabolic parameters and cardiovascular risk factors in patients with newly diagnosed type 2 diabetes mellitus. Diabetes Res Clin Pract. 2010;88:71-5.

10. Katakami N, Kaneto H, Matsuhisa M, Shimomura I, Yamasaki Y. Effects of glimepiride and glibenclamide on carotid atherosclerosis in type 2 diabetic patients. Diabetes Res Clin Pract. 2011;92:e20-2.

11. Zinman B, Wanner C, Lachin JM, et al. Empagliflozin, cardiovascular outcomes, and mortality in type 2 diabetes. N Engl J Med. 2015;373:2117-28.

12. Neal B, Perkovic V, Mahaffey KW, et al. Canagliflozin and cardiovascular and renal events in type 2 diabetes. N Engl J Med. 2017;377:644-57.

13. Kosiborod M, Cavender MA, Fu AZ, et al. Lower risk of heart failure and death in patients initiated on sodium-glucose cotransporter-2 inhibitors versus other glucose-lowering drugs. Circulation. 2017;136:249-59.

14. Suzuki M, Honda K, Fukazawa M, et al. Tofogliflozin, a potent and highly specific sodium/glucose cotransporter 2 inhibitor, improves glycemic control in diabetic rats and mice. J Pharmacol Exp Ther. 2012;341:692-701.

15. Kaku K, Watada H, Iwamoto Y, et al. Efficacy and safety of monotherapy with the novel sodium/glucose cotransporter-2 inhibitor tofogliflozin in Japanese patients with type 2 diabetes mellitus: a combined phase 2 and 3 randomized, placebocontrolled, double-blind, parallel-group comparative study. Cardiovasc Diabetol. 2014;13:65.

16. Tanizawa Y, Kaku K, Araki E, et al. Long-term safety and efficacy of tofogliflozin, a selective inhibitor of sodium-glucose cotransporter 2, as monotherapy or in combination with other oral antidiabetic agents in Japanese patients with type 2 diabetes mellitus: multicenter, open-label, randomized controlled trials. Expert Opin Pharmacother. 2014;15:749-66.

17. Cefalu WT, Leiter LA, Yoon K-H, et al. Efficacy and safety of canagliflozin versus glimepiride in patients 
with type 2 diabetes inadequately controlled with metformin (CANTATA-SU): 52 week results from a randomised, double-blind, phase 3 non-inferiority trial. Lancet. 2013;382:941-50.

18. Ridderstråle M, Andersen RA, Zeller C, et al. Comparison of empagliflozin and glimepiride as add-on to metformin in patients with type 2 diabetes: a 104-week randomised, active-controlled, doubleblind, phase 3 trial. Lancet Diabetes Endocrinol. 2014;2:691-700.

19. Nauck MA, Rohwedder K, Del Prato S, et al. Dapagliflozin versus glipizide as add-on therapy in patients with type 2 diabetes who have inadequate glycemic control with metformin. Diabetes Care. 2011;34:2015-22.

20. Downes MJ, Bettington EK, Gunton JE, Turkstra E. Triple therapy in type 2 diabetes; a systematic review and network meta-analysis. Peer J. 2015;3:e1461.

21. Lozano-Ortega G, Goring S, Bennett HA, Bergenheim K, Sternhufvud C, Mukherjee J. Network meta-analysis of treatments for type 2 diabetes mellitus following failure with metformin plus sulfonylurea. Curr Med Res Opin. 2016;32:807-16.

22. Lee CM, Woodward M, Colagiuri S. Triple therapy combinations for the treatment of type 2 diabetes-a network meta-analysis. Diabetes Res Clin Pract. 2016;116:149-58.

23. Blonde L, Stenlöf K, Fung A, Xie J, Canovatchel W, Meininger G. Effects of canagliflozin on body weight and body composition in patients with type 2 diabetes over 104 weeks. Postgrad Med. 2016;128:371-80.

24. Ohta A, Kato H, Ishii S, et al. Ipragliflozin, a sodium glucose co-transporter 2 inhibitor, reduces intrahepatic lipid content and abdominal visceral fat volume in patients with type 2 diabetes. Expert Opin Pharmacother. 2017;18:1433-8.

25. Tobita H, Sato S, Miyake T, Ishihara S, Kinoshita Y. Effects of dapagliflozin on body composition and liver tests in patients with nonalcoholic steatohepatitis associated with type 2 diabetes mellitus: a prospective, open-label, uncontrolled study. Curr Ther Res Clin Exp. 2017;87:13-9.

26. Matsuba R, Matsuba I, Shimokawa M, Nagai Y, Tanaka Y. Tofogliflozin decreases body fat mass and improves peripheral insulin resistance. Diabetes Obes Metab. 2018;20:1311-5.

27. Blacher J, Agnoletti D, Protogerou AD, et al. Aortic stiffness, inflammation, denutrition and prognosis in the oldest people. J Hum Hypertens. 2012;26:518-24.

28. Izumi M, Manabe E, Uematsu S, Watanabe A, Moritani T. Changes in autonomic nervous system activity, body weight, and percentage fat mass in the first year postpartum and factors regulating the return to prepregnancy weight. J Physiol Anthropol. 2016;35:26. 